\title{
Awareness and Impact of International Normalized Ratio Monitoring in Ongoing Anticoagulation Therapy in Cardiac Patients
}

\author{
Mriganka Pal' ${ }^{1}$, Suresh Bandari ${ }^{*}$, Scott Gier $^{2}$, Chandra Sekar ${ }^{3}$ \\ ${ }^{1}$ Department of pharmacy practice and Pharmaceutics, St. Peter's Institute of Pharmaceutical Sciences, Warangal, Telangana -506001, \\ INDIA. \\ ${ }^{2}$ Wyandot Memorial Hospital, Upper Sandusky, Ohio 43351, USA. \\ ${ }^{3}$ College of Pharmacy, University of Findlay, Findlay, Ohio 45840, USA.
}

\begin{abstract}
Objectives: The objectives of the current study is to determine the extent of awareness and the level of understanding about INR testing among patients undergoing oral anti-coagulation (OAT) therapy and to determine whether their INR values are within therapeutic range as measured by Coagucheck on-site INR. Results: For our study we interviewed 205 patients, and enrolled 90 patients that lasted for 6 months. Patient population recruited in the study comprised of 53 males and 37 females and INR was determined on-spot for these patients by CoaguChek. Majority of patients enrolled in this study were in the age group 20-59. Results indicate that more patients lacked awareness than aware of the importance of INR. Among 63 patients who were unaware about the significance of regular INR testing, $74.60 \%$ get their INR tested only if asked by a physician, $22.22 \%$ get their INR tested on a monthly basis and $3.17 \%$ get their INR checked every week. Whereas, among the 27 patients who were aware, $70.37 \%$ gets their INR tested on a weekly basis, $25.92 \%$ gets tested on a monthly basis and only $3.70 \%$ do it only if asked by a physician. Results also revealed that a greater proportion of the patients in the "lack of aware group" have INR values that are beyond the therapeutic range. Of the total of 7 Bleeding events reported by patients in this study, 6 were reported by patients who belonged to the "lack of aware group". Conclusion: Our study clearly demonstrates that ignorance regarding the significance of INR is clearly associated with increased incidence of bleeding. Patient education in conjunction with on-site INR testing by the pharmacist will both save lives and improve therapeutic outcome..
\end{abstract}

Key words: Warfarin, Anticoagulation, Monitoring, INR, On-site, Coagucheck. Indian hospital

\section{INTRODUCTION}

Acenocoumarol and warfarin are the most widely prescribed oral anticoagulants $(\mathrm{OAC})$ that are used to manage the thromboembolic complications. In hospital setting OAC's are the drug of choice for various indications like nosocomial deep vein thrombosis, pulmonary embolism, myocardial infarction, atrial fibrillation, unstable angina, rheumatic heart disease, vascular surgery, prosthetic heart valve, extracorpuscular circulation, hemodialysis and defibrillation syndrome. ${ }^{1,2}$ The prophylactic use of OAC therapy underlies the pharmacological basis to counteract thromboembolism and its associated complications in cardiac patients. ${ }^{3}$ The inherent definite risk of adverse outcomes associated with these OAC's mandates routine therapeutic drug monitoring that needs to be undertaken to ensure safe and effective therapy.

Measurment of International normalized ratio (INR) is an established integral part of anti-coagulation therapy that is used to determine the therapeutic dosage regimen required for an individual with a particular disease state. ${ }^{4-6}$ INR levels also show considerable intra-subject variability. Oral anticoagulants are routinely used to manage cardiac patients in India. As majority of patients reside in poor unsanitary condi-
DOI: 10.5530/ijopp.10.1.3

Address for correspondence: Dr. Suresh Bandari, Department of Pharmaceutics, St. Peter's Institute of Pharmaceutical Sciences, Hanamkonda, Warangal 506001 , Telangana State, INDIA.

Phone no: 9642880200 E-mail: reachbandari@gmail. com

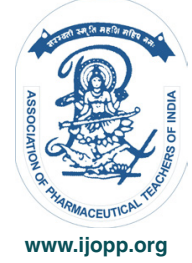


tions, many of them are simultaneously being treated with antibiotics such as metronidazole, macrolides, cotrimoxazole, and antifungals like fluconazole and miconazole. Many of these medications are capable of interacting with acenocoumarol and warfarin, thereby altering their clinical efficacy. Apart from medications, alcohol consumption (binge drinking) also increases the metabolism of oral anti-coagulant $\mathrm{t}^{1,2}$ thereby decreasing their effectiveness. As fate of anti-coagulation therapy is affected by many factors, it becomes critical to regularly monitor the INR value for optimum therapeutic outcome, especially in patients seen at outpatient departments. Patient's knowledge on the importance of INR and their awareness on monitoring INR is the major and significant determining factor that will lead to improved therapeutic management. ${ }^{5}$ The prime objective of this study is to determine patient awareness and to assess their level of understanding about routine INR testing and to determine how many of those patient's INR fall in the therapeutic range.

\section{METHODOLOGY}

A prospective cross-sectional pilot study was carried out in a cardiac center stationed at Warangal for the duration of 6 months. This study was approved by the human Ethical Committee of Mahatma Gandhi Memorial Hospital, Kakatiya Medical College, Warangal, Telangana, India.

During this study, 205 patients were interviewed and those fulfilling the inclusion criteria of this investigation were enrolled into the study. Inclusion criteria considered for the study included patients who are on on-going OAT, patients above age 12 of either gender. Exclusion criteria considered for the study included patients diagnosed with non-cardio embolic or non-thromboembolism conditions and patients who are less than 12 years in age. For each patient following information was collected in predesigned forms that included - demographic information such as age, sex, weight, address as well as family history of hypertension, diabetes mellitus, social habits, diagnosis, concomitant medication, past medication profile, anti-coagulation drug prescribed, and duration of OAT, hematological finding of previous visits and the questionnaire to understand the level of INR awareness.

Patients attending the outpatient department at the cardiac center were interviewed about the frequency of their INR testing, knowledge about their ongoing medication therapy and importance of regular INR testing and whether they had been counseled and educated on these points, complaints during their ongoing therapy, any missed doses or any switching of drugs by the prescriber in last 6 months. Real time on spot INR was determined with use of the device Coagucheck by Roche for every person enrolled in this study.

Data was analyzed using Microsoft excel and SPSS version 22. The results were analyzed and expressed as numerical values and as percentage. Adverse events were determined for causality using Naranjo's scale and clinician's advice.

\section{RESULTS}

During the course of this study, 90 patients were enrolled and interviewed for awareness on routine INR testing and their realtime INR value was measured. The study cohort registered consisted of 37 (41\%) females and $53(59 \%)$ males (Figure 1). Based on age the study cohort was classified into three categories - adults (1945 years), middle age (46-59 years) and geriatric (60 or above years).

Distribution profile for the three groups were as follows-Adults $37.77 \%$, Middle age $44.44 \%$ and geriatric $17.77 \%$. Minimum and maximum age of the patient population was 19 and 89, respectively, with a mean of 67.4 and standard deviation of 10.46. Minimum and maximum weight of the study population was 40 and $90 \mathrm{~kg}$, respectively.

The only anticoagulant prescribed to the study population was acenocoumarol, which was used alone in 68 $(75 \%)$ patients and in combination with aspirin in the remaining $25 \%$ of the patients.

Among the 90 patients participating in this study, we found that 63 patients i.e. $(70 \%)$ were neither aware about their ongoing medication therapy nor the importance of the regular INR testing for them, while the remaining 27 patients i.e. (30\%) know about the treatment they were on and also knew about the significance of regular INR testing (Figure 2).

Out of 63 patients who lacked awareness about the regular INR testing, 47 patients $(74.60 \%)$ get their INR tested only if asked by a physician (OIAP), 14 patients $(22.22 \%)$ get their INR tested on a monthly basis and 2 patients $(3.17 \%)$ on a weekly basis. Among the 27 aware patients, 19 patients $(70.37 \%)$ gets their INR tested on a weekly basis, whereas 7 patients $(25.92 \%)$ gets tested on a monthly basis and only 1 patient $(3.70 \%)$ do it only if asked by a physician (Figure 3).

The clinically accepted therapeutic INR range of 2-4 was considered normal for the study population and was determined by the physician and pathologist. ${ }^{7}$ INR data obtained on-site by us revealed that there are 15 patients with INR $>4$, 46 patients with INR between 2-4, and 29 patients with INR $<2$ are more at risk to develop a 


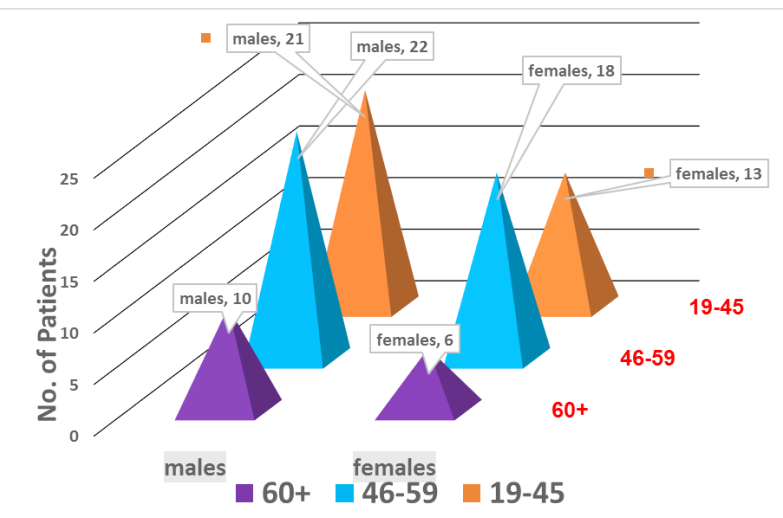

Figure 1 : Population distribution based on age and gender.

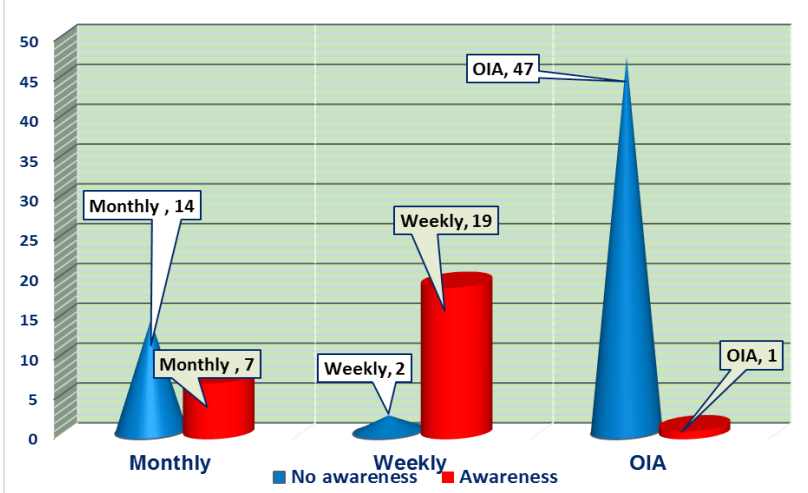

Figure 3: Frequency of INR testing in Aware and Non Aware population.

clot because of sub therapeutic dose of anticoagulant. We found that 3 and 4 bleeding incidents were reported in with INR cut off $>4$ and $>3$ respectively by the time of INR measurement.

Among the enrolled population $47.61 \%$ showed abnormal INR value and $52.31 \%$ showed INR value within the therapeutic range. When we compared the on-site INR values among lack of awareness group, it was observed that $66.66 \%$ population had abnormal INR values, whereas in awareness group $47.61 \%$ showed abnormal INR values as seen in Figure 4.

When we compared the distribution of the population with duration of OAT, following was observed, out of 90 patients enrolled 41 patients were on OAT for 6 months or less, 24 patients were between 6-12 months, 5 patients were between 12 months to 24 months and 20 patients were more than 24 months. Of the 41 patients who have had OAT for only 6 months, 23 patients showed abnormal INR value, out of 24 patients in 6-12 months 11 patients showed abnormal INR value. Similarly out of 5 patients between 12-24 months and

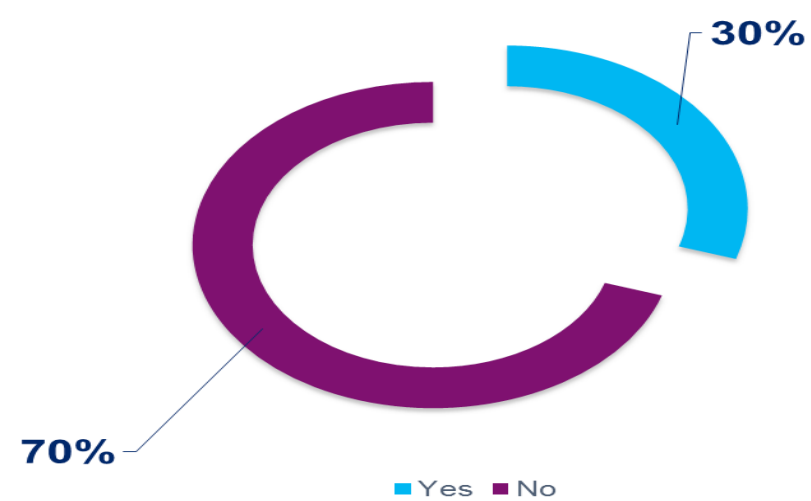

Figure 2: Pie-chart showing the awareness in the study population.

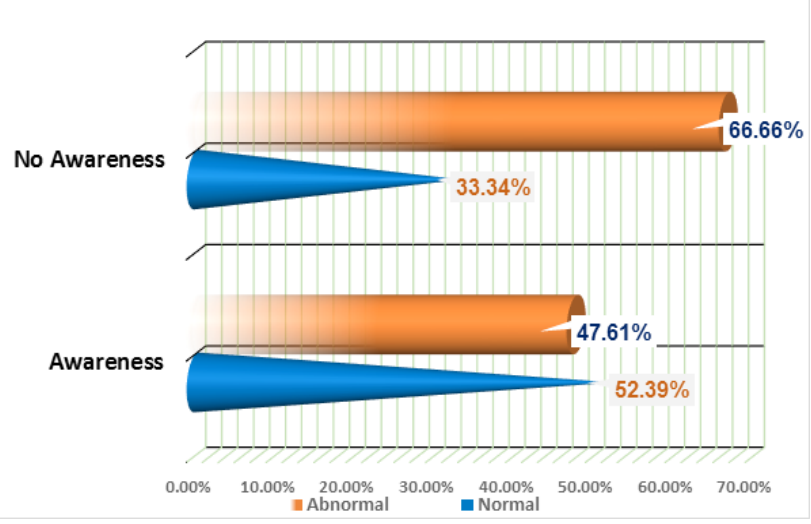

Figure 4: Normality and abnormality of INR value in aware and and unware population.

20 patients in more than 24 months, 2 and 7 patients showed abnormal values respectively.

\section{DISCUSSION}

The cohort registered in the study reveals that oral anticoagulation therapy is used in both males and females, with maximum number of users in the middle age group (46-59). In this study the middle age group is found to be predominant in comparison with geriatric/elderly group. ${ }^{3}$ According to Nadine R. Sahyoun et al., there is a high mortality rate among the adult age group. ${ }^{8}$

Acenocoumarol is extensively marketed in India and hence the only anticoagulant employed in patients who were enrolled into the study. ${ }^{9}$ This was used alone or in combination with aspirin depending upon patient's diagnosis done by the physician which was indicated and justified on case basis, as per standard clinical guidelines set for the use of anti-coagulants essential in drug therapy to treat multiple complications of cardio vascular diseases. Overall awareness about routine INR testing and its significance in OAT therapy was poor among registered patients. This could be attributed to 
the rural location and a lower literacy rate in the participant population in this study. The unaware patient population gets their INR values tested when a physician asked them during their visit which would be the bimonthly or quarterly basis. However, they should have rather been tested on weekly basis in initial phase of the therapy, at least once in every 4 weeks in the stable phase. During transition phase and start of concurrent medication therapy that interacts with warfarin metabolism, it is very important to closely monitor the INR values of the patient to circumvent risk of bleeding. ${ }^{10}$ Only $3.17 \%$ patients and $22.2 \%$ tested their INR values within the visit i.e. on weekly and monthly basis respectively. Among the aware population the routine INR testing was more appropriate, which included $70 \%$ on a weekly basis and $25 \%$ on a monthly basis and $3.7 \%$ only if asked by a physician. This data clearly reveals that routine INR testing significantly depends on the awareness about the oral anticoagulation therapy. The observation of unaware population showed $33.34 \%$ with normal INR value and $66.6 \%$ patients with abnormal INR values, while the aware population showed normal INR values in $52.39 \%$ and $47.61 \%$ with abnormal INR values, suggesting the impact of awareness and significance of routine INR testing by the patients.

The patients with INR value $>4$ showed $42.86 \%$ bleeding incidences, this may be due to lack of medication adherence and unawareness about regular INR testing. The study observation reveals that there are 57.14\% incidence of bleeding within normal range of INR value $2-4$. This could be probably due to concomitant medication and co-morbidities of the patients.

\section{CONCLUSION}

In a lower middle income country such as India, majority of the population may be unable to afford routine INR monitoring on the following two counts -

Cost involved in testing.

Time investment required to visit hospital laboratory for the blood draw.

Introduction of point of care INR testing kits, where pharmacist can determine INR value of the patient without a laboratory visit provides an appropriate solution for the second point.

While we realize that "INR kits" are still expensive from the Indian standards for these patients, third parties (whether it is government or insurance) realizing the value of health care savings will be able to subsidize the testing. As demand for instant INR kits increases, that should spur the development of local products priced for developing market because analysis of our results clearly indicate an association between "lack of knowledge on INR" and increased incidence of bleeding. Therefore patient education in conjunction with onsite INR testing by the pharmacist will both save lives and improve therapeutic outcome.

\section{ACKNOWLEDGMENT}

We sincerely thank our guide Dr. Siddharth A Prasad, Interventional Cardiologist (Honorary) for providing constant support, affectionate encouragement, and precious advice.

We would like to express our deep gratitude to Mr. T. Jaypal Reddy Chairman St.Peter's Institute of Pharmaceutical Sciences for his constant support and encouragement towards such innovative initiatives.

\section{CONFLICT OF INTEREST}

The authors declare no conflict of interest.

\section{ABBREVIATION USED}

INR: International normalized ratio; OAT: Oral anticoagulation therapy; OAC: Oral anticoagulants; OIAF: Only if asked by the physician.

\section{REFERENCES}

1. Online Document Bpacnz. INR testing, Nov 2010. Available from http://www. bpac.org.nz/BT/2010/November/inr.aspx

2. Vijay S, krishnappa G, Amirali B, Neelathahalli kM. Anticoagulation Utilization Evaluation in a tertiary care teaching hospital; anobservational prospective study in medical in patients. Indian Journal of Pharmacy practice. 2015;8(2):10.5530.

3. Nasser S, Mullan J, Bajorek B. Challenges of older patients' knowledge about warfarin therapy. Journal of Primary Care and Community Health. 2011;1-10.

4. Karen F, Samuel ZG. A Patient's Guide to Taking Coumadin/Warfarin. Circulation. 2009;119:e220-e2.

5. A report by the Anticoagulation Self-Monitoring Alliance; Anticoagulation services and patient access to INR self-monitoring in the NHS in England: July 2014.

6. Jonas OI, Healey JS, Ezekowitz M, Commerford P, Alvaro Avezum et al. Variations in Etiology and Management of Atrial Fibrillation in a Prospective Registry of 15,400 Emergency Department Patients in 46 Countries: The RELY AF Registry. Circulation. 2014;113.005451.

7. Braunwald's Heart Disease, A textbook of cardiovascular medicine.10th ed. Philadelphia. Mann Zipes Libby Bonow. 2015.

8. Sahyoun NR, Lentzner H, Hoyert D, Robinson KN. Trends in Causes of Death Among the Elderly. Aging Trends; No.1. Hyattsville, Maryland: National Center for Health Statistics. 2001.

9. Gopalakrishnan S, Srinivasan N. Oral Anticoagulants: Current Indian Scenario. Chapter 90;410-3.

10. Laboratory Survival Guide The University of Texas Medical Branch.Available from https://www.utmb.edu/LSG/Pages/ORAL_ANTICOAG_THERAPY.aspx. 